\title{
Performance Study of Impact-Point Estimation Systems Using Two Types of Acoustic Sensor Arrangement Methods
}

\author{
Shin Hur ${ }^{1 D},{ }^{1}$ EunJoong Lee, ${ }^{1}$ Jongseong Won, ${ }^{2}$ Duck-Gyu Lee, ${ }^{1}$ Kyungjun Song, ${ }^{3}$ \\ and Kyusik Park ${ }^{2}$ \\ ${ }^{1}$ Department of Nature-Inspired Nano Convergence Systems, Korea Institute of Machinery and Materials, Daejeon, \\ (34103) 156, Republic of Korea \\ ${ }^{2}$ Department of software science, Dankook University, Jukjeon, (16890) 152, Republic of Korea \\ ${ }^{3}$ Department of Mechanical Engineering, Pusan National University, (46241) 63-2, Geumjeong-Ku, Busan, Republic of Korea
}

Correspondence should be addressed to Shin Hur; shur@kimm.re.kr

Received 22 July 2019; Revised 11 September 2019; Accepted 16 September 2019; Published 11 November 2019

Academic Editor: Paolo Bruschi

Copyright $\odot 2019$ Shin Hur et al. This is an open access article distributed under the Creative Commons Attribution License, which permits unrestricted use, distribution, and reproduction in any medium, provided the original work is properly cited.

In this study, a shooting experiment is carried out with an acoustic sensor array that can detect shock wave at high-sound pressures and withstand the shock wave of the bullet. Delta- and H-type of acoustic sensor units are constructed. The measured values of the temporal arrival differences estimated based on the distance between the acoustic sensors are then compared with the theoretical calculations according to the types of bullets and shooting distance. In addition, an impact-point estimation system (IPES) is fabricated, which comprises delta- and $\mathrm{H}$-type sensor units, a target, signal processing device, and an impact point estimation algorithm. The impact points on the target caused by the shooting experiment are compared with those calculated using the impact point estimation algorithm. Finally, the mean absolute error (MAE) between the actual and the calculated impact-point coordinates is calculated, and the performances of the delta- and H-type IPES are evaluated.

\section{Introduction}

When a bullet is fired from a gun owing to the occurrence of the deflagration inside it, the air around the bullet is compressed and the bullet generates a cone-shaped shock wave. The generated blast wave then propagates at the sonic velocity in the radial direction. As the shock wave is generated in a clear $\mathrm{N}$-shape within a short time interval, the position of the bullet can be identified based on the time difference of the arrival (TDoA) of the shock wave. Acoustic sensors capable of measuring shock wave signals must withstand sound pressures greater than $140 \mathrm{~dB}$. They must also exhibit uniform detection performance regardless of temperature and humidity changes. In conventional shooting exercises, whenever a shooter shoots, the person's visual acuity or the assisting telescope confirm the location at which the bullet has penetrated the target plate. However, it is difficult and time consuming to determine the location of an impact point because the shooter uses the naked eye or the telescope to determine the location of the impact point. Therefore, various countries, including the US, UK, Switzerland, Sweden, and others, have developed impact-point estimation systems (IPES), such as the location of miss and hit (LOMAH) system, to support shooting training programs. The IPES can measure the impact of a bullet and display the bullet's impact point in real time. The system allows shooters to identify the point of impact with a display at a nearby location, which can shorten the time of shooting training and improve the safety of the training program. Training commanders can also manage training information and perform real-time assessments to summarize the shooting results and monitor the progress of trainees. A typical IPES consists of a shock-wave detection unit based on an acoustic a sensor array, a signal processing device, an impact-point estimation algorithm, a computer, and a monitor. In general, the shock-wave detection unit possesses signal processing functions and detects the shock wave generated by the bullet. To accurately estimate the impact point, the placement method and number of acoustic sensors 


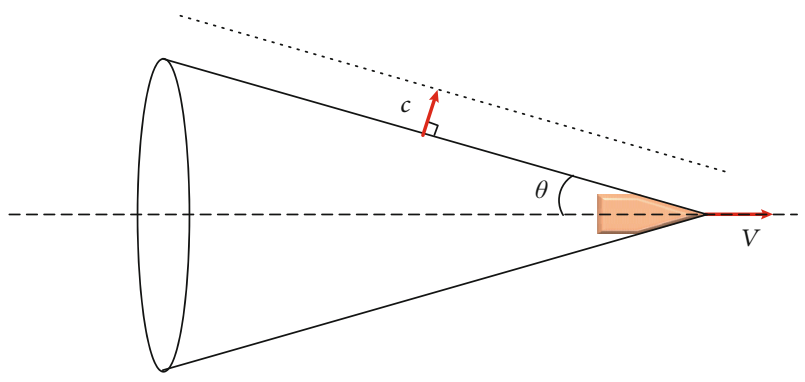

FIgURE 1: Generation of a cone-shaped shock wave for a bullet traveling with a velocity $\mathrm{V}$.

must be determined, and an effective impact-point estimation algorithm must be used. Previous studies related to this current investigation included theoretical and experimental studies of the shock wave and gunshot of the bullet generated by shooting, and the study of the time delay between the generation of the shock wave and the gunshot $[1,2]$. Furthermore, theoretical and experimental studies have been published that allow the detection of the position of a sniper with the use of fire detectors worn by soldiers [3, 4]. However, no prior studies compare the impact point performance with different bullet types and sensor arrangement methods using the IPES.

This study selects an acoustic sensor that is capable of sensing the shock wave of a bullet and compares the calculated and experimental values of TDoA of the shock wave caused by the distances between acoustic sensors. Furthermore, the IPES are fabricated with delta- and H-type of acoustic sensor units and the impact-point location of bullets on the target are obtained from an actual shooting experiment and compared with those calculated using the impactpoint estimation algorithm. The performances of the IPES are evaluated by comparing the errors of the impact-point coordinates between the shooting experiment and the impact-point estimation calculations.

1.1. Theory of Shock Wave. The speed of an object traveling faster than the speed of sound in air can be expressed using the Mach number, $M=V / c$, as the ratio of the speed of sound $c$ and the velocity of the object $V$. Figure 1 shows the shock wave generated by a bullet traveling at a velocity higher than the speed of sound from the compression of the air. This shock wave has a conical shape with an angle $\theta$ relative to the path of the traveling object, and the wave propagates in the vertical direction of the shock wave at the speed of sound [5].

In this case, the propagation angle $\theta$ of the shock wave is equal to $\arcsin (1 / M)$. The shock wave measured through an acoustic sensor has a clear $\mathrm{N}$-shape. The time interval $T$ from the highest to the lowest points of the $\mathrm{N}$-shape is expressed as follows [1]:

$$
T=1.82\left(\frac{d}{c}\right)\left(\frac{M x}{l}\right)^{1 / 4}
$$

where $d$ is the diameter of the bullet, $x$ is the vertical distance between the trajectory of the bullet and the acoustic sensor,

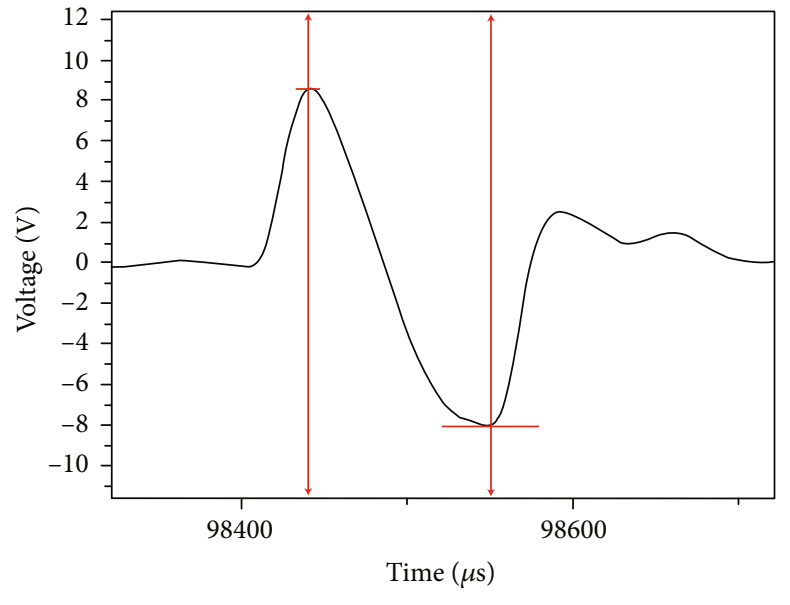

FIgURE 2: N-shape form of shock wave signal.

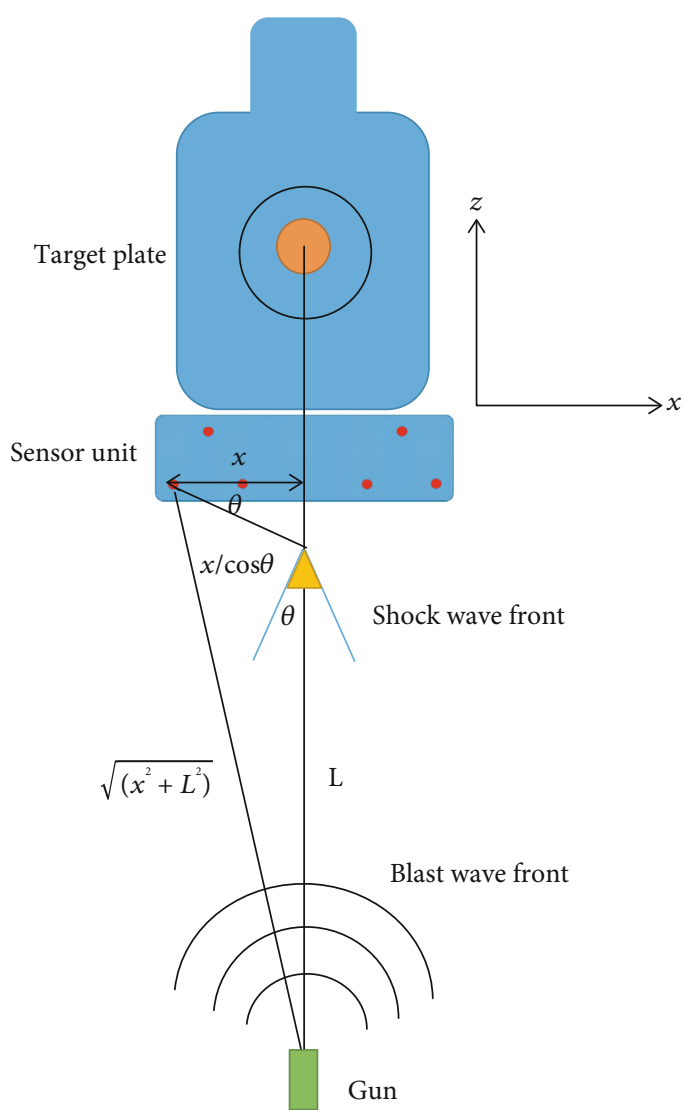

FIgURE 3: Schematic explaining the calculation of the shock and blast wave arrival times.

and $l$ is the length of the bullet. The speed of sound $c$ is expressed according to $331.5+0.6 \times t$, indicating that the temperature $t$ has a significant impact on the formation of the shock wave. Figure 2 shows a shock wave with a typical $\mathrm{N}$-shape. The time interval $T$ of the shock wave was measured as the peak-to-peak interval of the measured voltage shock wave signal. 


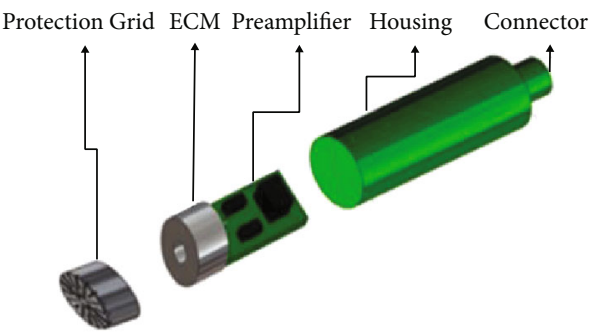

(a)

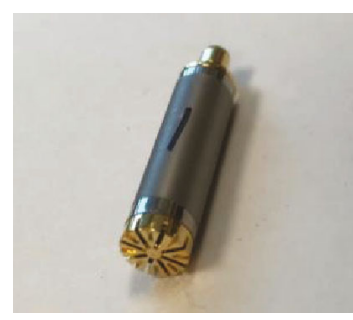

(b)

Figure 4: Acoustic sensor. (a) Schematic of structure and constituent components. (b) Photograph of acoustic sensor.

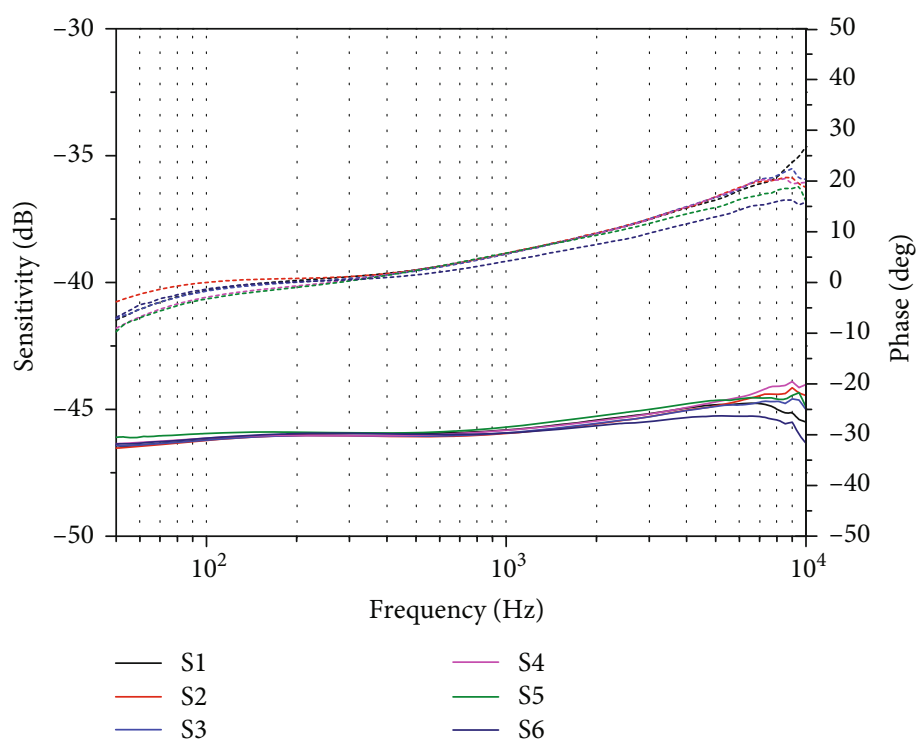

FIGURE 5: Sensitivity (solid lines) and phase (dotted lines) measurements obtained from the acoustic sensors.

Figure 3 shows a schematic for the calculation of the shock and blast wave arrival times. The distance between each acoustic sensor and the impact point can be expressed as $x$, the travel distance from the shock wave generated in the path of the bullet to the acoustic sensor as $x / \cos \theta$, and the travel time as $x /(\mathrm{c} \cdot \cos \theta)$. In this case, the distance from the time of shooting to the shock wave detection time by the acoustic sensor is expressed as $L-x \tan \theta$ using the shooting distance $L$. The corresponding travel time is $(L-x \tan \theta) / V$. The sum of the travel time of the bullet from the time of shooting and the travel time of the shock wave that reaches the acoustic sensor is determined as $((L-x \tan \theta) / V)+$ $x /(c \cdot \cos \theta)$. Using this expression, the shock wave arrival times at the six sensors can be calculated for an arbitrary shooting distance. The travel distance of the blast wave from the shooting point to the acoustic sensor can be expressed as $\sqrt{\left(x^{2}+L^{2}\right)}$, and the corresponding travel time is expressed as $\sqrt{\left(x^{2}+L^{2}\right)} / c$. When the shooting takes place at a distance of $100 \mathrm{~m}$, the shock wave arrival time is $138 \mathrm{~ms}$ at the temperature of $20^{\circ} \mathrm{C}$, the bullet velocity is $920 \mathrm{~m} / \mathrm{s}$, the bullet diameter is $7.62 \mathrm{~mm}$, the bullet length is $15 \mathrm{~mm}$, and the vertical distance $x$ between the bullet path and the sensor is $1 \mathrm{~m}$. The time difference of arrival (TDoA) between the shock
TABLE 1: Measurement results of the acoustic sensor.

\begin{tabular}{lc}
\hline Sensitivity & $-46.0 \mathrm{~dB}$ relative to $1 \mathrm{~V} / \mathrm{pa} \pm 3 \mathrm{~dB}$ \\
\hline Frequency response & $\pm 2 \mathrm{~dB}, 100 \mathrm{~Hz}$ to $10 \mathrm{kHz}$ \\
& $\pm 3^{\circ}, 100 \mathrm{~Hz}$ to $3 \mathrm{kHz}$ \\
Phase response & $\pm 5^{\circ}, 3 \mathrm{kHz}$ to $5 \mathrm{kHz}$ \\
\hline
\end{tabular}

wave and the blast wave measured by the acoustic sensor, i.e., $(L-x \tan \theta) / V)+x /(c \cdot \cos \theta)-\sqrt{\left(x^{2}+L^{2}\right)} / c$, increases as the shooting distance increases [1].

\section{Results and Discussion}

2.1. Experimental Measurements. A typical muzzle explosion produces shock waves with a sound pressure level $(\mathrm{SPL}) \geq 140 \mathrm{~dB}$. Thus, acoustic sensors used for measuring these shock waves must be able to withstand these sound pressures [6]. Therefore, several fire tests were conducted on various acoustic sensors. Based on these tests, the acoustic sensor that was capable of withstanding shock waves was selected. As shown in Figure 4(a), the structure of the 


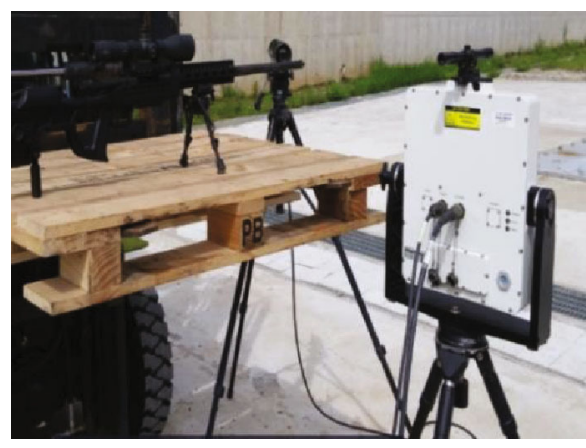

(a)

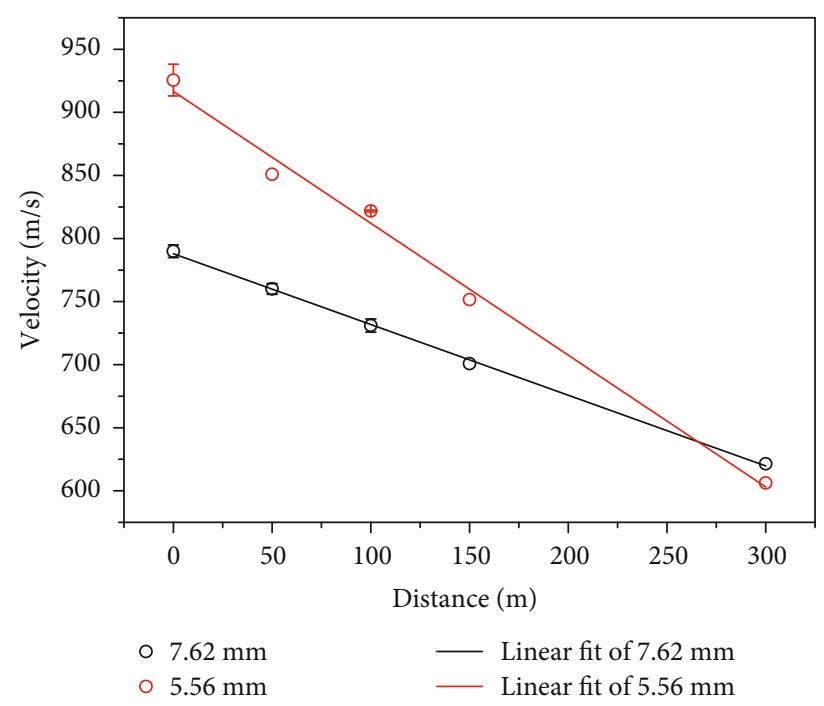

(b)

FIGURE 6: Bullet velocity measurement setup. (a) Doppler radar equipment. (b) Bullet velocity measurements as a function of the shooting distance.

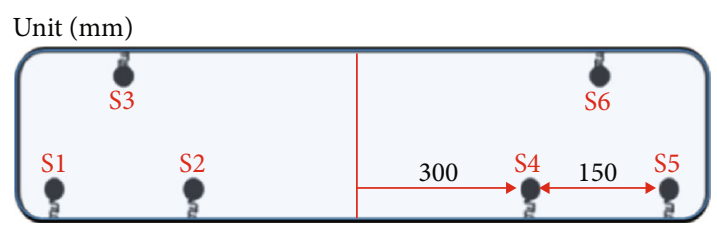

(a)

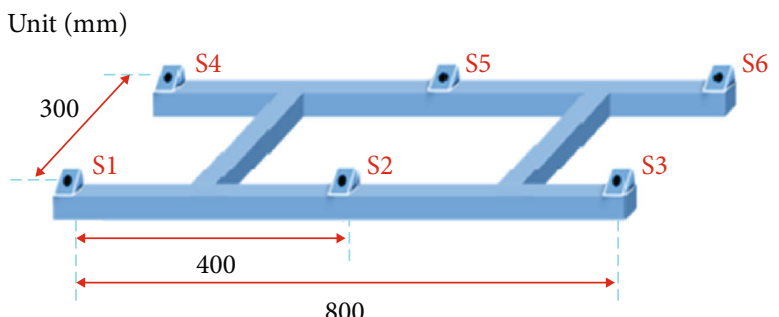

(b)

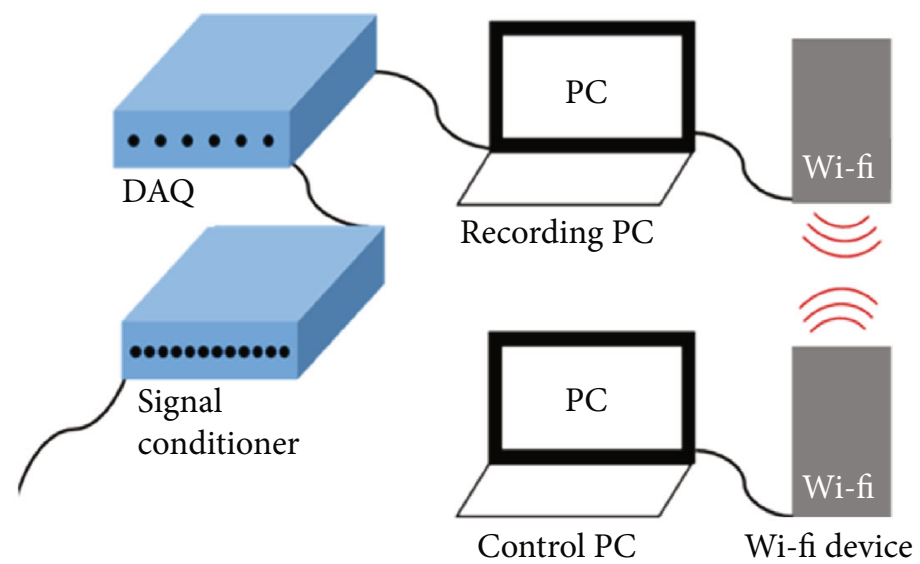

(c)

FIgURE 7: Schematic of the acoustic sensor units. (a) Delta-type sensor unit. (b) H-type sensor unit. (c) Data processing setup.

acoustic sensor is composed of a protection grid, an electret condenser microphone (ECM), a preamplifier, housing, and an SMB connector. Figure 4(b) shows the acoustic sensor (HJ06, SISPIA Co., Korea). Each of the acoustic sensors should also have uniform sensitivity and phase characteristics to predict accurately the impact point of the bullet. The sensitivity and phase characteristics of the acoustic sensors were measured in an anechoic chamber according to the standard measurement method. The sensitivity of the acoustic sensors was approximately $-46.0 \mathrm{~dB}$ in the frequency band of $100 \mathrm{~Hz}$ to $10 \mathrm{kHz}$, and the phase was approximately within $\pm 3^{\circ}$ at $1.0 \mathrm{kHz}$. Figure 5 shows the sensitivity and 

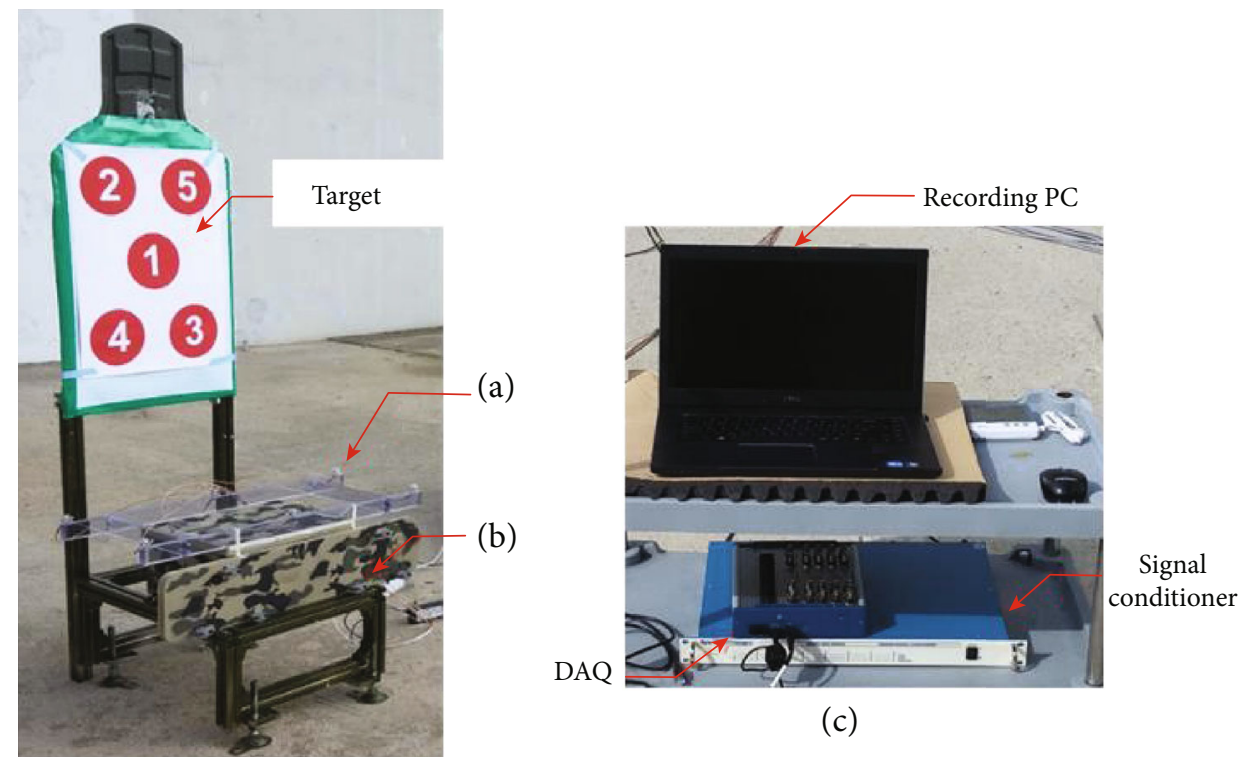

(c)

Figure 8: Test setup of the impact-point estimation system (IPES). (a) H-type sensor unit. (b) Delta-type sensor unit. (c) Data processing setup.

phase measurement outcomes from the six acoustic sensors, thus indicating the uniform sensitivity and phase characteristics with respect to frequency. The performance specifications of the acoustic sensors are listed in Table 1. The IPES based on the delta- and H-type sensor units was constructed with the use of the six acoustic sensors with uniform sensitivity and phase value.

To measure the shock wave of a bullet and to test the performance of the IPES, an experiment was performed in an actual shooting test site. Bullets with diameters equal to $5.56 \mathrm{~mm}$ and $7.62 \mathrm{~mm}$ were used. Prior to the experiment, the bullet velocity was measured at $50 \mathrm{~m}, 100 \mathrm{~m}, 150 \mathrm{~m}$, and $300 \mathrm{~m}$ from the shooting point with a speed measuring device (Doppler radar SL-528PE, Denmark) based on the Doppler effects at the shooting position. Figure 6 shows the bullet velocity as a function of distance for bullets with diameters equal to $5.56 \mathrm{~mm}$ and $7.62 \mathrm{~mm}$. For locations near the shooting distance, the $5.56 \mathrm{~mm}$ bullet with a diameter of $5.56 \mathrm{~mm}$ is faster than that the bullet with a diameter of $7.62 \mathrm{~mm}$. At the shooting distance of $260 \mathrm{~m}$, the $7.62 \mathrm{~mm}$ bullet with the diameter of $7.62 \mathrm{~mm}$ is faster.

Figure 7 shows a schematic of the experimental setup for the shock wave detection units. Figures 7 (a) and 7(b) show the delta- and H-type of acoustic sensor units with six acoustic sensors in each of them. In addition, Figure 7(c) shows the data processing setup that contains a signal conditioner (PCB-480E09), a data acquisition system (DAQ, NI USB6366), LabVIEW (NI Signal Express) software, and a recording PC. In addition, a wireless communication device (MAP5020) operating based on Wi-Fi technology and control PC is used to collect and control the data acquisition of generated shock waves at long distances. The sampling rate of the shock wave measurement is $200 \mathrm{kHz}$, and the data transmission rate is $20 \mathrm{kHz}$. The shooting distances are 100 $\mathrm{m}$ and $200 \mathrm{~m}$, and the bullet diameters are $5.56 \mathrm{~mm}$ and $7.62 \mathrm{~mm}$.

Figure 8 shows the test setup of the IPES for shock wave measurement and impact-point identification. Based on the actual shooting and the six acoustic sensors placed in the delta-type and H-type sensor units, shock waves are collected at the shooting distances of $100 \mathrm{~m}$ and $200 \mathrm{~m}$. Figures 9(a) and 9(b) show the shock and blast waves measured at the shooting distances of $100 \mathrm{~m}$ and $200 \mathrm{~m}$, respectively. These results correspond to the shooting experimental results of the $7.62 \mathrm{~mm}$ bullet with the delta type sensor unit. These outcomes show that the blast waves arrive after the arrival of the shock waves. Figures 9(c) and 9(d) show the shock waves measured by the six acoustic sensors at the shooting distances of $100 \mathrm{~m}$ and $200 \mathrm{~m}$, respectively, using the same conditions. Herein, S1-S6 represent the six acoustic sensors whose arrangements are shown in Figure 7.

The arrival time of the blast wave can be simply calculated as $\sqrt{\left(\mathrm{x}^{2}+\mathrm{L}^{2}\right)} / c$. Figure 10 shows the experimental and calculated time delays between the shock and blast waves according to the shooting distance measured from the deltatype sensor unit. Based on this figure, it is confirmed that the time delay of the shock wave increases as the shooting distance increases, and the error range of the experimental values is very small. However, the difference between the experimental and calculated values increases as the shooting distance increases. A maximum difference of $7.0 \%$ is obtained for a distance of $200 \mathrm{~m}$. This appears to be caused because the theoretical equation cannot consider the nonlinear characteristics of the flight of the bullet, such as the drag, which occurs in longdistance flights as the shooting distance increases.

Use of the point of impact at the instant the bullet penetrated the target in the actual shooting test, allows the calculation of the actual coordinates from the center of the arrayed 


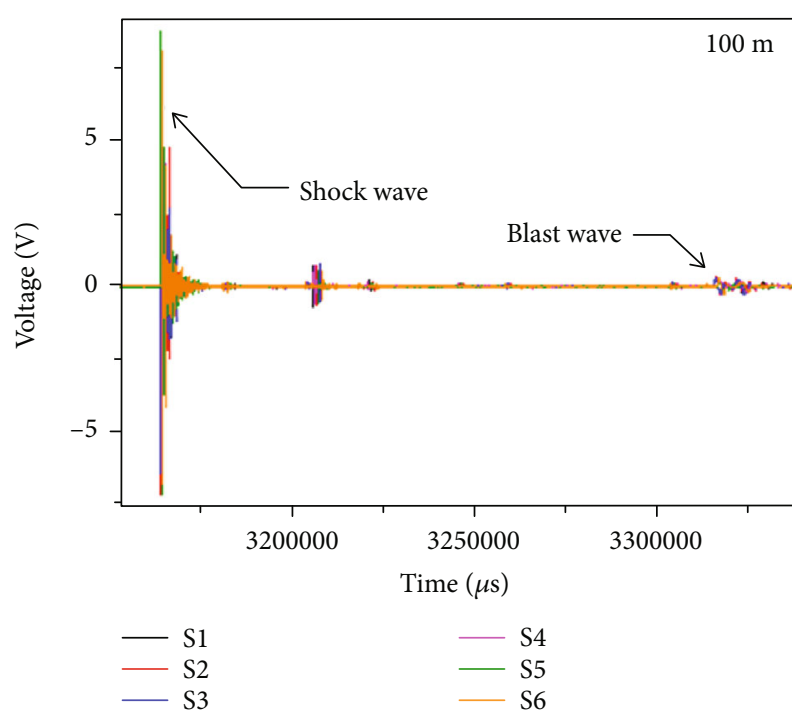

(a)

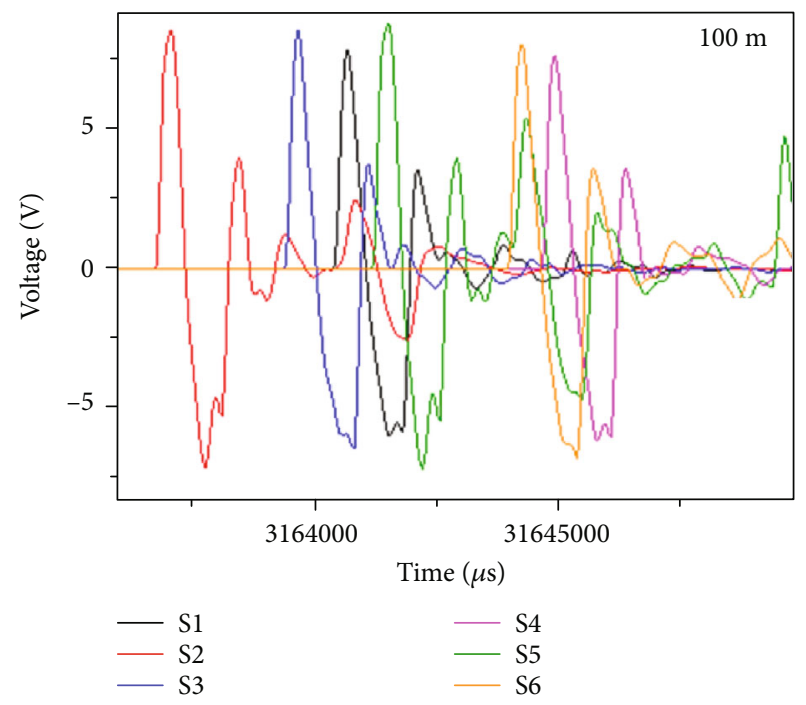

(c)

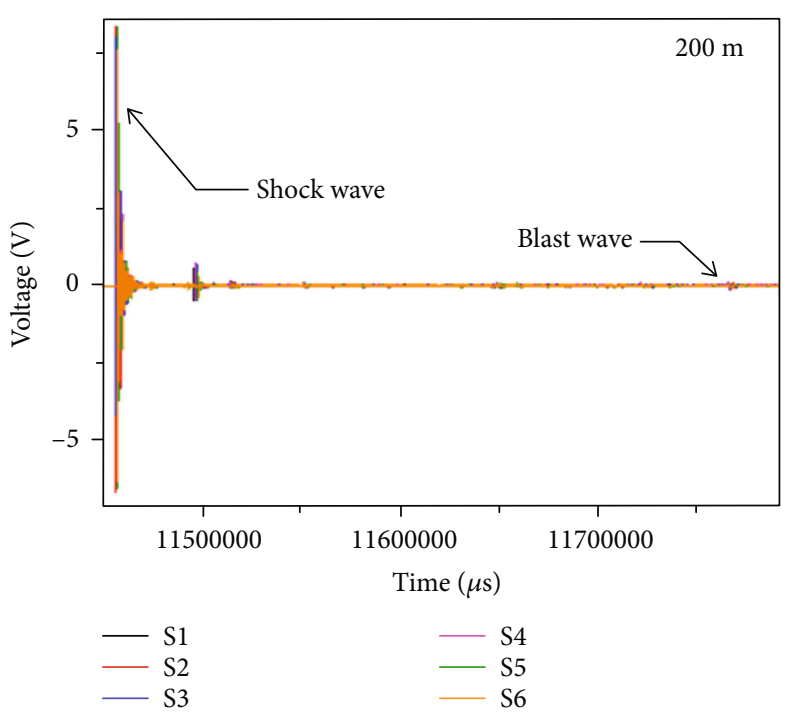

(b)

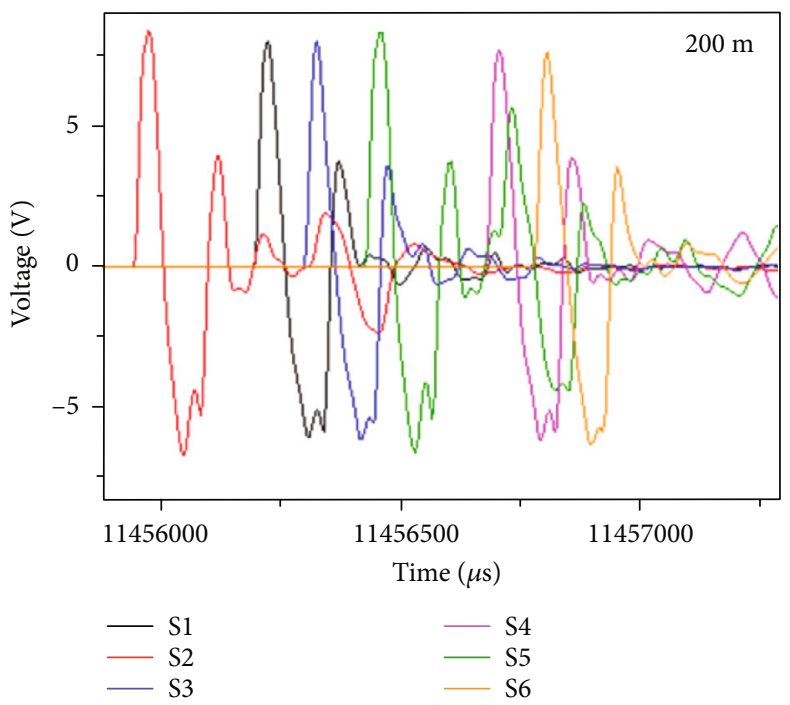

(d)

FIGURE 9: Results of the shooting experiment with a delta-type sensor unit and a $7.62 \mathrm{~mm}$ bullet. (a, b) Shock waves and blast waves measured according to the shooting distance. (c, d) Shock waves the showing time differences among the acoustic sensors.

sensor. To estimate the coordinates of the actual impact point, the time delay of the shock wave arriving at each sensor must be measured. In Figure 7, the delta-type sensor unit contains two separate groups of sensors at the left and the right sides, while the H-type sensor unit has two separate groups at the front and rear. Each group comprises three sensors each. Based on this configuration, the arrival times among the sensors can be calculated. Figures 11(a) and 11(b) show the differences between the experimental and calculated TDoA values of the shock wave with bullet diameters of $5.56 \mathrm{~mm}$ and $7.62 \mathrm{~mm}$ according to the shooting distance in the cases of delta-and H-type sensor units. The TDoA value refers to the difference in the arrival time of the shock wave with respect to the arrival times at other acoustic sensors, and it is based on the signal that arrives first among all the acoustic sensor arrays. The delta-type sensor unit exhibits a deviation difference of $\sim 2.1 \%$, while the H-type sensor unit yields a deviation difference of $14.2 \%$. This indicates that the delta-type sensor unit yields a higher accuracy in the estimation of the arrival time of the shock wave.

2.2. Impact-Point Estimation Algorithm. The impact-point estimation algorithm proposed by Levanon [7] defined the time of arrival (ToA) of the bullet shock wave at each microphone using simultaneous equations and estimated the impact point using least-squares estimation, such as the Gauss-Newton (GN) algorithm.

Figure 12 shows the shock wave occurrence time point $B$, microphone position $S_{k}$, actual impact point $\mathrm{H}$, and horizontal incidence angle $\alpha$, when the bullet penetrates the 


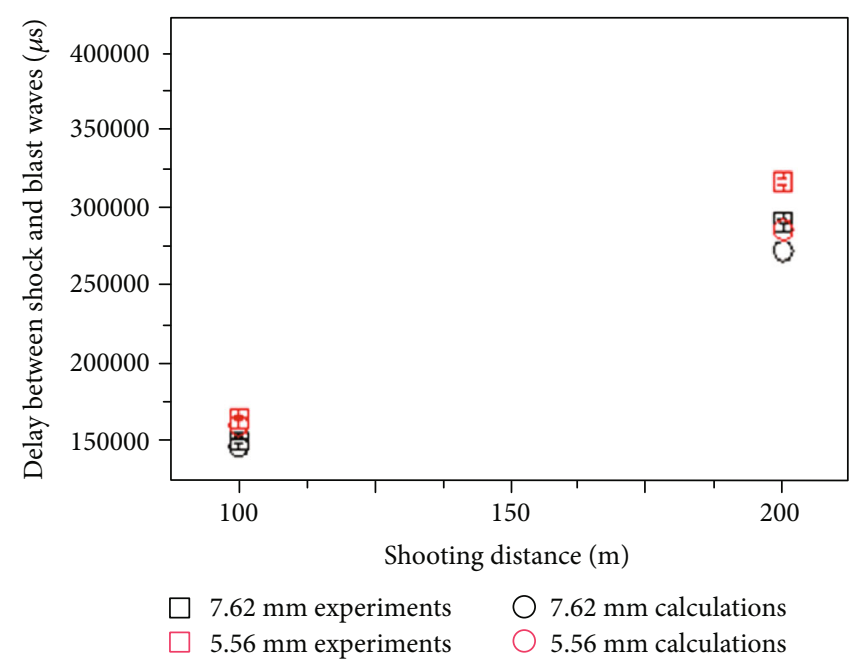

FIgURE 10: Experimental and calculated time delays between the shock and blast waves.

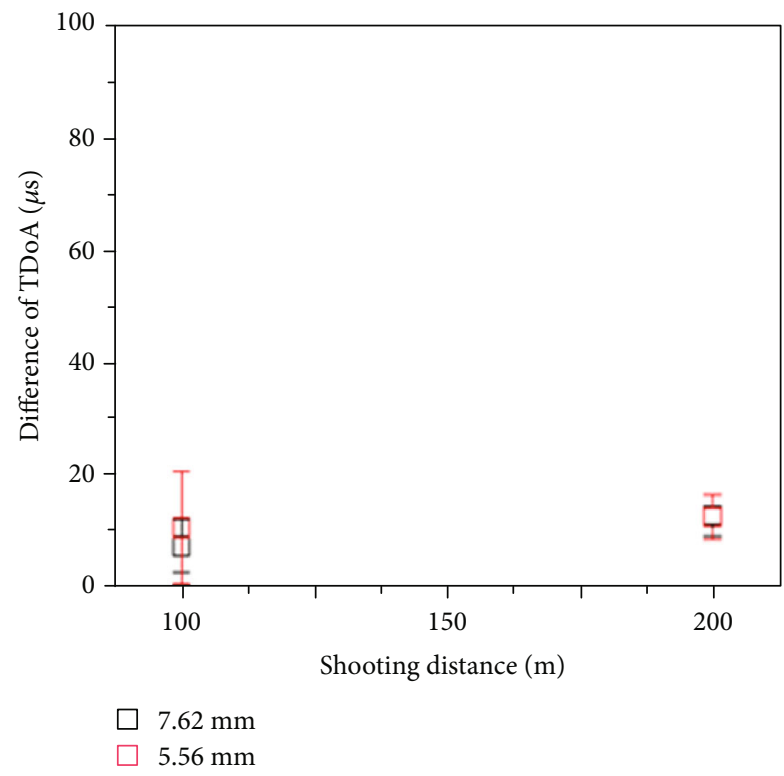

(a)

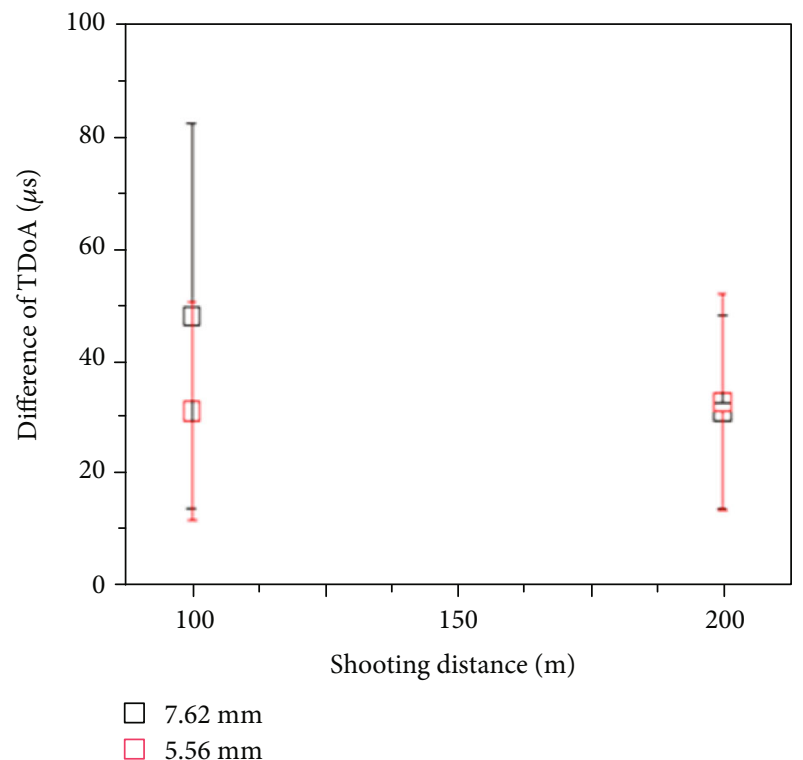

(b)

Figure 11: Differences between the experimental and calculated values of the TDoA between the sensors according to the shooting distance $(100 \mathrm{~m}, 200 \mathrm{~m})$. (a) Delta- and (b) H-type sensor units. The error bars represent the standard deviations from three measurements obtained with two types of bullets.

target. If the ToA at which the shock wave that occurs at $\mathrm{B}$ and arrives at $\mathrm{S}_{k}$ is defined as $t_{k}, t_{k}$ can be expressed according to Equation (2).

$$
\begin{aligned}
t_{k}= & t_{o f f s e t}-\frac{1}{V}\left[\left(x-x_{k}\right) \sin \alpha-y_{k} \cos \alpha\right] \\
& +\sqrt{\left[\left(x-x_{k}\right) \cos \alpha+y_{k} \sin \alpha\right]^{2}+\left(h-h_{k}\right)^{2}} \times \sqrt{\frac{1}{C^{2}}-\frac{1}{V^{2}}}
\end{aligned}
$$

where $t_{\text {offset }}$ is the common delay time for all the microphones and is expressed as $t_{\text {offset }}=-y_{0} / V, c$ is the speed of sound, $V$ is the velocity of the bullet, and $y_{0}$ is the position of the signal that reaches the microphone. Nonlinear equations are defined in the number of microphones using the arrival time and position of each microphone. The equation listed above contains six variables. Specifically, $x$ and $h$ are impact points, and $\alpha$ is the horizontal incidence angle. Therefore, to calculate the simultaneous equations, six or more equations, i.e., six or more microphone signals 


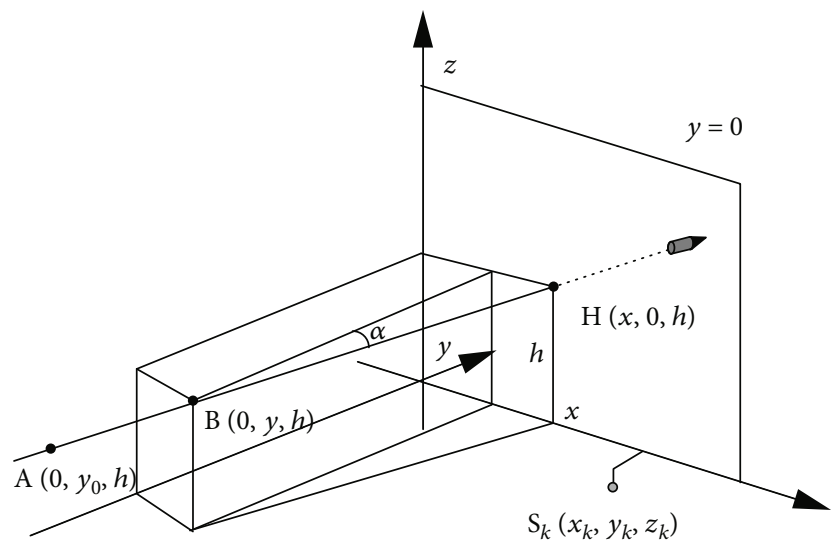

FIgURE 12: Geometry of target.

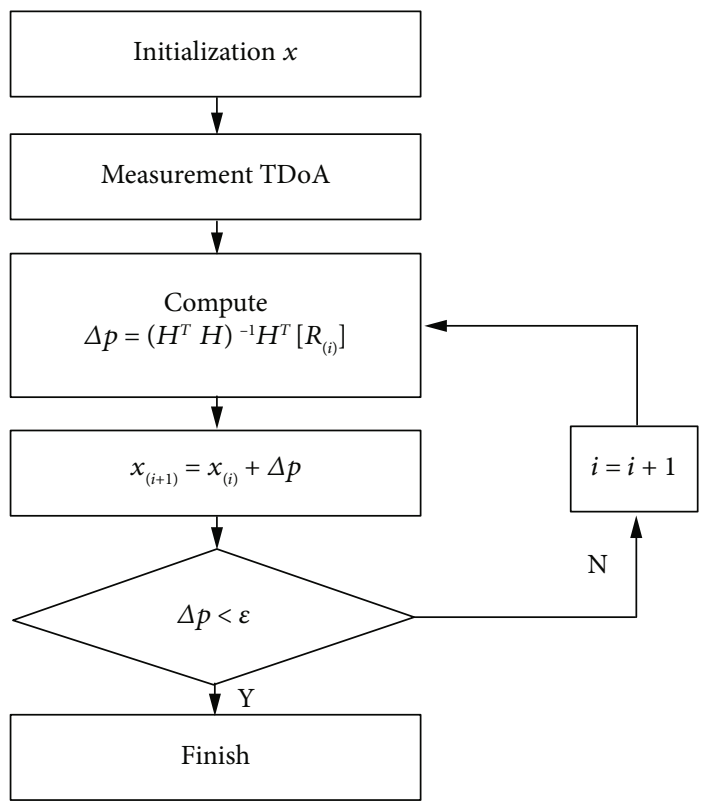

FIGURE 13: Flow chart of the impact-point estimation algorithm.

are required. In this study, signals were acquired from acoustic sensors using delta- and H-type sensor units.

The method proposed by Levanon [7] uses the ToA method. Compared with the TDoA method, this method produces significantly different operation results when there are ToA measurement errors [8], including the instances at which time synchronization problems occur. Therefore, the impact points of the delta- and H-type sensor units were estimated using the TDoA-based impact-point estimation algorithm proposed by Won and Park [9].

Figure 13 shows a flow chart of the process used for the impact-point estimation algorithm. The algorithm designates initial values (impact point, speed of sound, and bullet velocity), performs iterative operations using the GN algorithm, and completes the iterative operations and displays the results when the variation of the operation result decreases below the set value. $\mathrm{H}$ denotes the partial differential of $\chi$, and $\mathrm{R}(\mathrm{i})$ denotes the difference between the measured time difference and the calculated time difference. $\Delta p$ represents the amount of change in $\mathrm{x}$ and $\mathrm{y}$ at each iteration.

Even though 20 rounds were fired in the shooting experiment, 13 datasets were obtained because seven rounds missed the target. Table 2 lists the shooting conditions and measured coordinates as well as the coordinates calculated using TDoA for the 13 datasets acquired from the shooting experiment. Furthermore, the data for the shooting distance of $200 \mathrm{~m}$ included only three datasets. At this time, the $x$-axis coordinate represents the horizontal coordinate and the $z$-axis coordinate represents the vertical coordinate. The center point of the target used in the experiment is converted into the coordinates $(0,900)$.

Figure 14 shows the measured impact-point coordinates as well as the calculated impact-point coordinates using the delta- and H-type sensor units. Compared to those estimated 
TABLE 2: The measured and the calculated impact-point coordinates.

\begin{tabular}{|c|c|c|c|c|c|c|c|c|}
\hline \multirow[t]{2}{*}{ Test times } & \multirow[t]{2}{*}{ Shooting distance $(\mathrm{m})$} & \multirow[t]{2}{*}{ Bullet diameter (mm) } & \multicolumn{2}{|c|}{$\begin{array}{c}\text { Measured } \\
\text { coordinates }(\mathrm{mm})\end{array}$} & \multicolumn{2}{|c|}{$\begin{array}{c}\text { Calculated } \\
\text { Coordinates } \\
(\mathrm{mm}), \text { Delta-type }\end{array}$} & \multicolumn{2}{|c|}{$\begin{array}{l}\text { Calculated } \\
\text { coordinates } \\
(\mathrm{mm}), \text { H-type }\end{array}$} \\
\hline & & & $\mathrm{X}$ axis & $\mathrm{Z}$ axis & $\mathrm{X}$ axis & $\mathrm{Z}$ axis & $\mathrm{X}$ axis & $\mathrm{Z}$ axis \\
\hline 1 & \multirow{10}{*}{100} & \multirow{5}{*}{7.62} & 60.0 & 843.0 & 68.5 & 844.5 & 48.7 & 818.5 \\
\hline 2 & & & -68.0 & 1050.0 & -63.5 & 1048.6 & -76.4 & 1030.3 \\
\hline 3 & & & 126.0 & 669.0 & 128.3 & 667.0 & 111.8 & 648.9 \\
\hline 4 & & & -36.0 & 648.0 & -33.0 & 649.3 & -43.6 & 623.3 \\
\hline 5 & & & 87.0 & 1063.0 & 95.6 & 1060.0 & 77.2 & 1032.4 \\
\hline 6 & & \multirow{5}{*}{5.56} & 35.0 & 965.0 & 39.6 & 962.3 & No data & No data \\
\hline 7 & & & -47.0 & 1058.0 & -38.4 & 1048.2 & -53.5 & 1023.0 \\
\hline 8 & & & 118.0 & 712.0 & 112.4 & 709.4 & 105.1 & 689.5 \\
\hline 9 & & & -20.0 & 625.0 & -10.2 & 624.1 & -22.6 & 596.9 \\
\hline 10 & & & 93.0 & 922.0 & 101.4 & 908.6 & 77.0 & 887.6 \\
\hline 11 & \multirow{3}{*}{200} & \multirow{2}{*}{7.62} & 113.0 & 765.0 & 116.1 & 770.7 & 99.4 & 743.2 \\
\hline 12 & & & 231.0 & 1072.0 & 228.2 & 1070.5 & 208.9 & 1032.0 \\
\hline 13 & & 5.56 & 55.0 & 709.0 & 52.7 & 712.2 & 41.9 & 686.4 \\
\hline
\end{tabular}

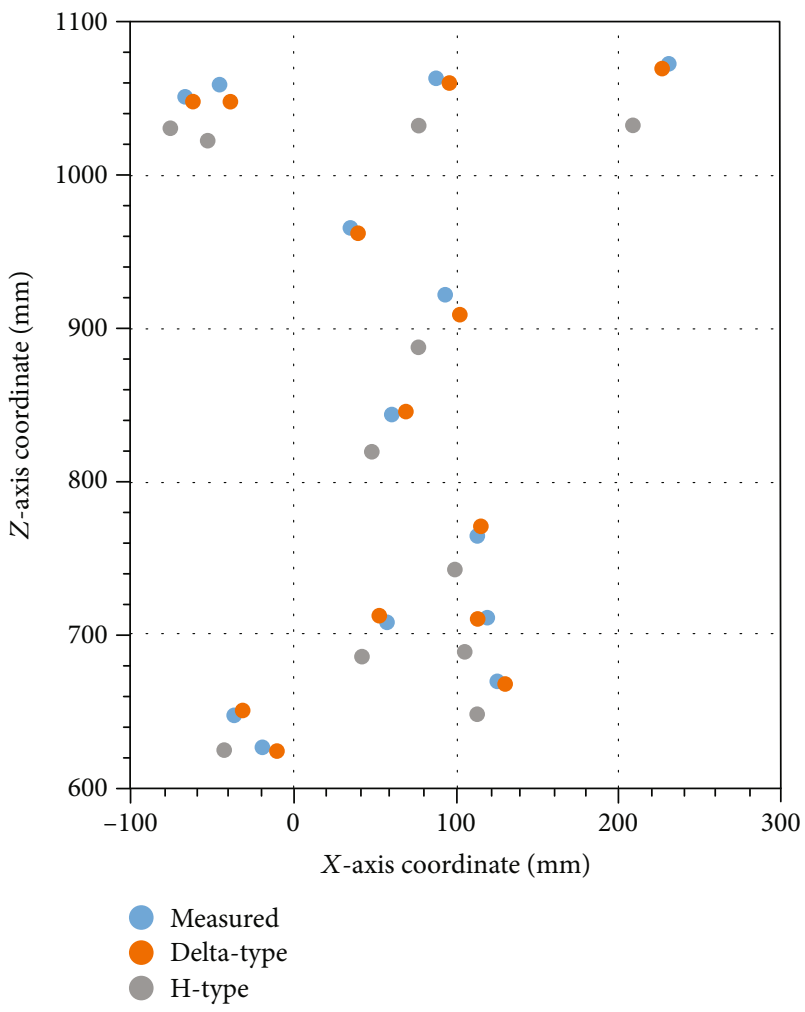

FIGURE 14: The measured and calculated impact-point coordinates.

for the delta-type sensor unit, the calculated coordinates estimated for the H-type sensor unit are slightly biased to the direction at the bottom-left. In addition, the H-type sensor unit has difficulties the determination of the measured impact point in the upward-downward directions because all six acoustic sensors are present on the same plane, as shown in Figure 15. As the impact-point estimation algorithm

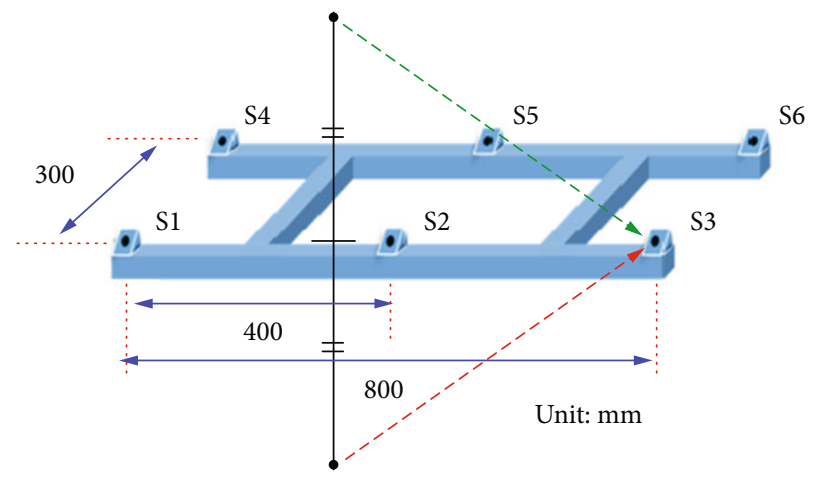

Figure 15: Schematic shows a potential problem with the same signal in an H-type sensor unit.

performs operations by designating the target center as the initial value, an actual bullet that passes through the bottom of the acoustic sensors can be determined as being positioned above the acoustic sensors. To address this problem, it seems necessary to place additional acoustic sensors or to adjust the positions of the existing acoustic sensors.

Conversely, the delta-type sensor unit is capable of determining the impact point in the upward-downward directions, and thus avoids the problem identified above. The mean absolute error (MAE) between the measured coordinates and the calculated coordinates of impact-point position is estimated with Equation (3).

$$
\mathrm{MAE}=\frac{\left(\sum_{i=1}^{n}\left|m_{i}-c_{i}\right|\right)}{n}
$$

where $\mathrm{m}$ is the measured coordinate, $c$ is the calculated coordinate and $n$ is the number of data points. 
TABLE 3: Mean absolute error according to bullet type.

\begin{tabular}{lccccc}
\hline $\begin{array}{l}\text { Shooting } \\
\text { Distance } \\
(\mathrm{m})\end{array}$ & $\begin{array}{c}\text { Bullet } \\
\text { Diameter } \\
(\mathrm{mm})\end{array}$ & $\mathrm{X}$ axis MAE $(\mathrm{mm})$ & Z axis MAE $(\mathrm{mm})$ & X axis MAE $(\mathrm{mm})$ & Z axis MAE $(\mathrm{mm})$ \\
\hline \multirow{2}{*}{100} & 5.56 & 7.40 & 5.88 & 9.5 & 30.00 \\
& 7.62 & 5.38 & 1.84 & 10.26 & 23.92 \\
200 & 5.56 & 2.30 & 3.2 & 13.1 & 22.60 \\
& 7.62 & 2.95 & 3.6 & 17.85 & 30.95 \\
\hline
\end{tabular}

Table 3 shows the MAE values of the delta- and H-type sensor units for different bullet diameters. In the case of the shooting distance of $100 \mathrm{~m}$, the MAE value of the delta-type is relatively smaller than the MAE value of the $\mathrm{H}$-type. Accordingly, it is considered that the delta-type of impact prediction performance is better. In the case of the H-type sensor unit, the reason why the MAE value of the $\mathrm{Z}$ axis is larger than the MAE value of the $\mathrm{X}$ axis is attributed to the fact that the problem arises because the sensor is arranged in the $\mathrm{X}$ axis as mentioned above. Furthermore, given that the MAE value of the bullet with a diameter of $7.62 \mathrm{~mm}$ is relatively smaller than that for the bullet with a diameter of $5.56 \mathrm{~mm}$, it is considered that the impact prediction performance of the bullet with a diameter of $7.62 \mathrm{~mm}$ is superior. In the case of the shooting distance of $200 \mathrm{~m}$, the MAE value of the delta-type is also relatively smaller than the MAE value of the H-type. However, given that the MAE value is calculated using only three shooting datasets, the reliability of the MAE value cannot be confirmed.

\section{Conclusions}

This study presents the first performance evaluation outcomes on the accuracy of the IPES according to two types of acoustic sensor arrangement methods. First, the acoustic sensor suitable for measuring shock waves was selected, and the various acoustic sensors were then tested. Subsequently, acoustic sensors with uniform sensitivity and phase characteristics were used in the shooting experiment, and it was confirmed that they had almost identical response characteristics for shock wave measurements. An IPES was constructed with the use of delta-type and H-type sensor units with the selected acoustic sensors, and shock waves were collected by performing shooting experiments at the shooting distances of $100 \mathrm{~m}$ and $200 \mathrm{~m}$, and with two types of bullets with the diameters of $5.56 \mathrm{~mm}$ and $7.62 \mathrm{~mm}$. By comparing the experimental and calculated TDoA values of the shock wave for delta-type and H-type sensor units, the TDoA values of the delta type sensor unit exhibited a low difference deviation of $2.1 \%$. The coordinates of the impact point were obtained from actual shooting tests and the impact-point estimation algorithm based on TDoA datasets for the deltaand $\mathrm{H}$-type sensor units. Because the MAE values of the delta-type sensor unit were relatively smaller than those for the H-type sensor unit, it was considered that the performance of the delta-type impact-point estimation was better than that of the H-type unit. It is expected that this study will contribute to the improvement of the accuracy of the IPES based on the acoustic sensor arrangement method.

\section{Data Availability}

The data used to support the findings of this study are available from the corresponding author upon request.

\section{Conflicts of Interest}

The authors declare that there is no conflict of interest regarding the publication of this paper.

\section{Funding}

This study was supported by the KIMM Project (UD0910) of the Civil Military Technology Cooperation Program (16-PDSS-02) and the Industrial Strategic Technology Development Program) (10078310, MO9500, Development of High Speed Multi-pass DTP System) funded by the Ministry of Trade, Industry \& Energy (MOTIE, Korea).

\section{References}

[1] R. C. Maher, "Modeling and signal processing of acoustic gunshot recordings," in 2006 IEEE 12th Digital Signal Processing Workshop \& 4th IEEE Signal Processing Education Workshop, pp. 257-261, Teton National Park, WY, USA, September 2006.

[2] R. C. Maher and S. R. Shaw, "Deciphering gunshot recordings," in Audio Engineering Society Conference: 33rd International Conference: Audio Forensics-Theory and Practice, Audio Engineering Society, 2008.

[3] J. George and L. M. Kaplan, "Shooter localization using a wireless sensor network of soldier-worn gunfire detection systems," Journal of Advances in Information Fusion, vol. 8, no. 1, pp. 15-32, 2013.

[4] G. L. Duckworth, J. E. Barger, S. H. Carlson et al., "Fixed and wearable acoustic counter-sniper systems for law enforcement," in Proceedings Volume 3577, Sensors, C3I, Information, and Training Technologies for Law Enforcement. International Society for Optics and Photonics, Boston, MA, USA, 1999.

[5] T. Mäkinen and P. Pertilä, "Shooter localization and bullet trajectory, caliber, and speed estimation based on detected firing sounds," Applied Acoustics, vol. 71, no. 10, pp. 902-913, 2010.

[6] B. G. Horman, Sound Suppressors 101, American Rifleman, 2013.

[7] N. Levanon, "Acoustic hit indicator," 1999, U.S. Patent No. $5,920,522$. 
[8] J.-W. Park, J.-H. Park, S.-H. Song, and T.-K. Sung, "Comparisons of error characteristics between TOA and TDOA positioning in dense multipath environment," Transactions of the Korean Institute of Electrical Engineers, vol. 58, no. 2, pp. 415-421, 2009.

[9] J. Won and K. Park, "Impact point estimation system of the rifle based on time difference of arrival method using microphone array," Journal of the Acoustical Society of Korea, vol. 37, no. 4, pp. 206-214, 2018. 


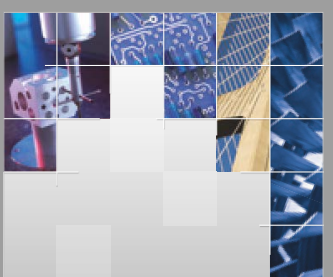

\section{Enfincering}
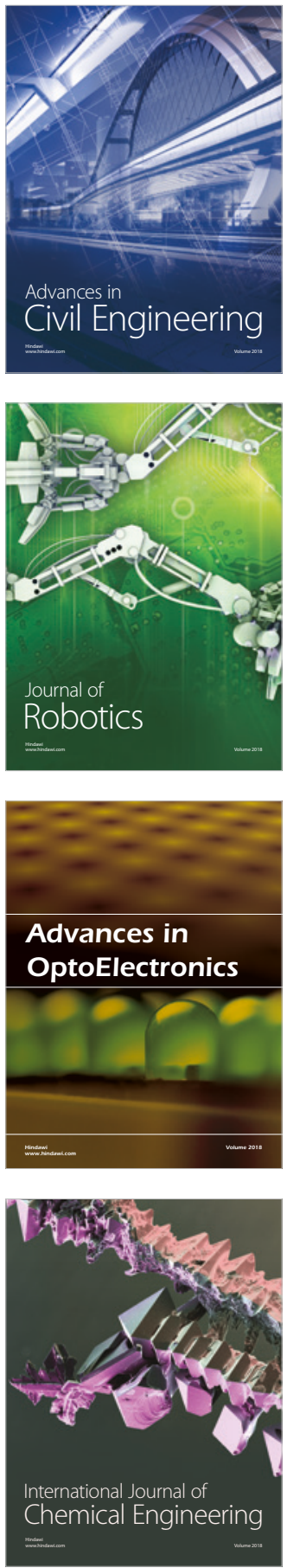

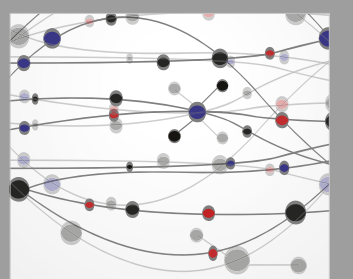

\section{Rotating \\ Machinery}

The Scientific World Journal

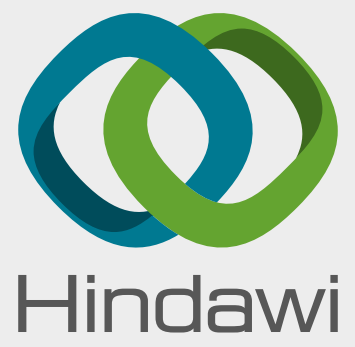

Submit your manuscripts at

www.hindawi.com
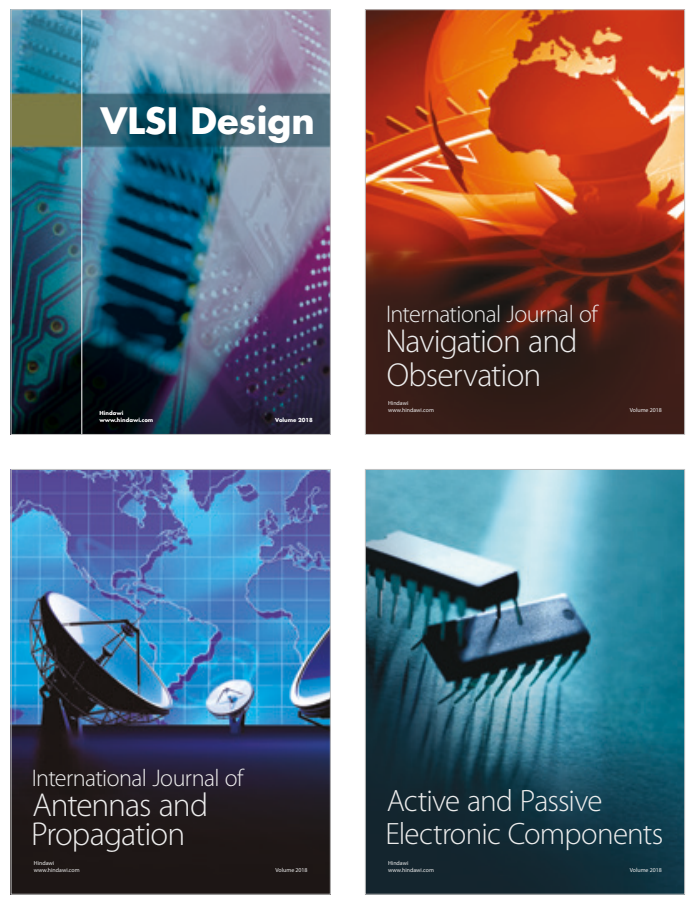
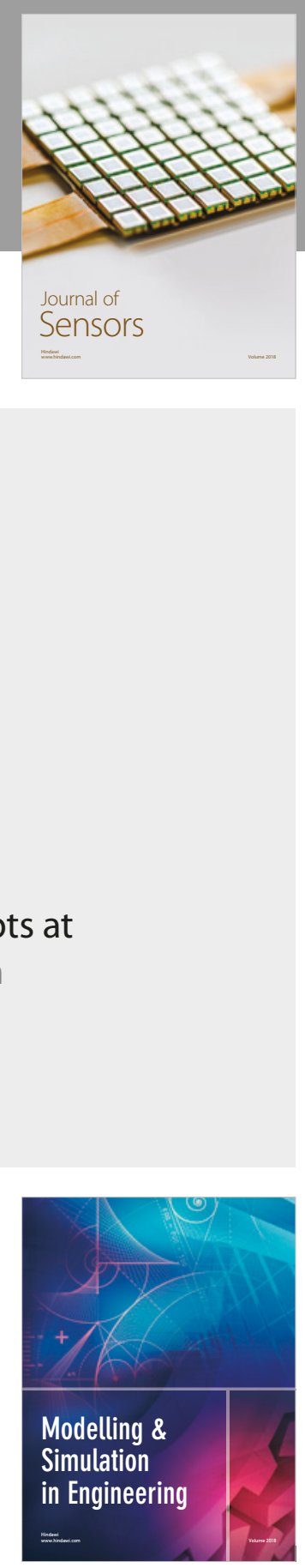

\section{Advances \\ Multimedia}
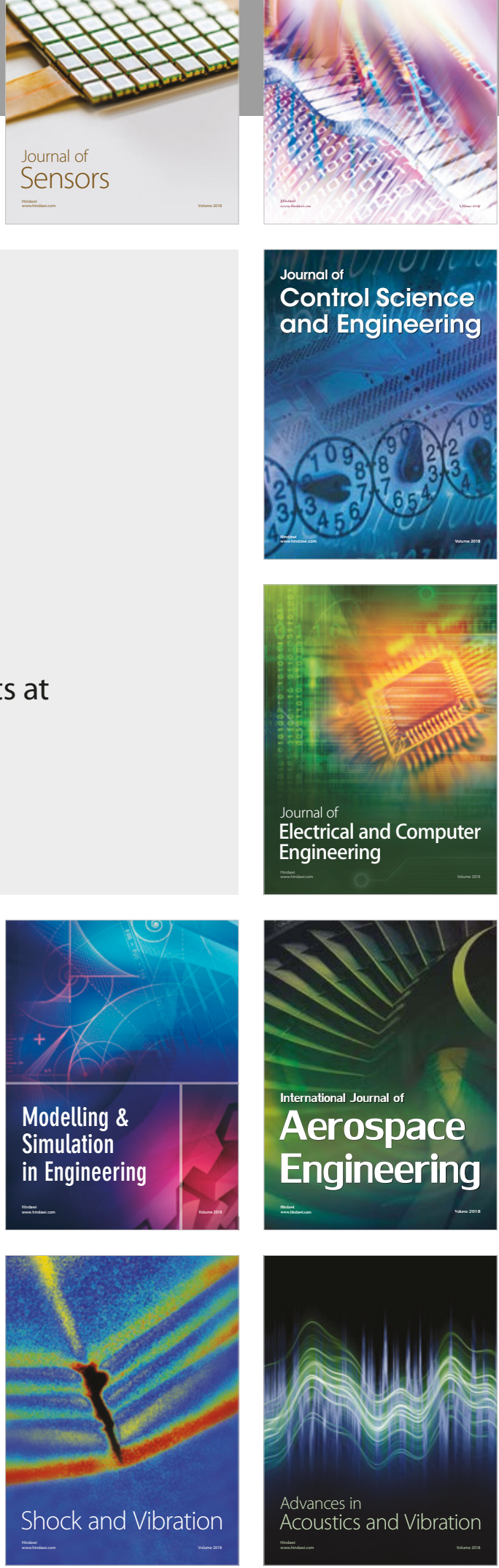